\title{
IP-Based TV TeChnologies, Services, AND MULTIDISCIPLINARY APPLICATIONS
}

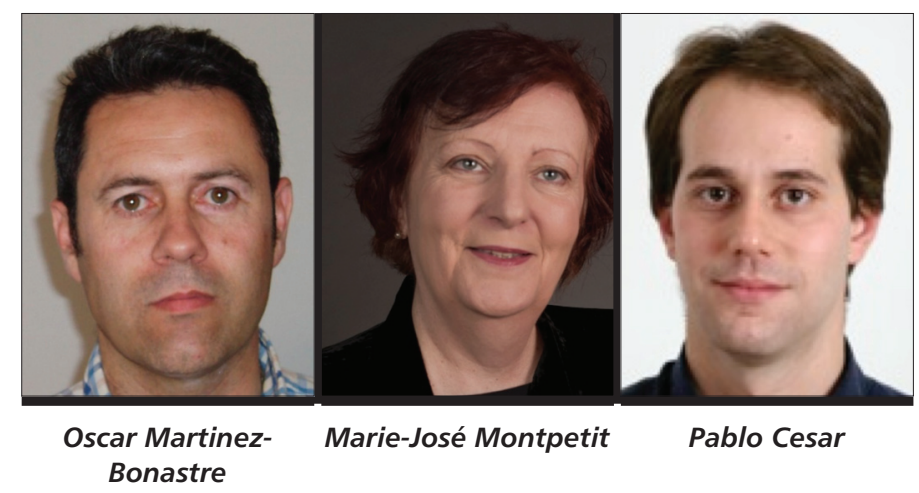

Join the online discussion group for this Feature Topic here: http://community.comsoc.org/forums/commag-features-and-series

$\mathrm{T}$ he move to Internet Protocol (IP)-based content delivery services has challenged the television industry by allowing high-quality television content to be delivered using the Internet, wired and wireless, private and public. The new convergence paradigm is already playing out its disruptive role as "television" is now associated with both a personal and social experience using an ecosystem of devices. The unidirectional flow of content from an operator to a device has thus morphed into a much richer combination of real-time on-demand web and user generated content complementing traditional programming.

With more and more platforms such as tablets, connected TVs, smartphones, andtraditional and IP-enabled settop boxes available for content distribution and consumption, the TV experience is geo-localized, and time and place shifted, and combines video streaming, social networking, micro-blogging, and widgets. And it is wireless and mobile, with phenomenal growth predicted in the next few years.

Hence, while critics have announced the death of television, with this special issue we intend to show that not only is television alive, but it has given life to many novel architectures for networks and middleware, improvements to the reliability of the connected information nodes, new distribution models to maximize the performance in wireless environments, as well as user-centric experience research, social television, transmedia storytelling, and user interface research. Essentially, research on television has moved from focusing on the traditional linear delivery model to studying comprehensive end-to-end and top to bottom strategies. It has moved away from the current design silos and the balkanization of devices and services, and now addresses the comprehensive and intelligent use of the multiscreen.
As a result, television convergence research is being renewed to investigate the advances that will be necessary in networking and user experience. Ubiquitous television provides a significant challenge for the next generation networks, especially wireless, the future viewing devices, the "screens," the software platforms, and the user interfaces. Significant questions need to be answered: how to manage content delivery networks, protect commercial and personal content, design efficient distribution architectures, enhance visual perception, and maximize the quality of experience. The articles in this Feature Topic (FT) focus on the recent changes in the Internet as the new television delivery medium and how these changes reshape the IP-based television of the future.

The three articles chosen after an extensive review (which have been selected out of 24 submissions in a tworound peer review process) embody how the changes in television consumption of the recent past have challenged platforms and infrastructure. They focus on mainly two areas, a storytelling platform and channel switching performance, which are of great importance for the future of the "new television."

In the first article, entitled "Storisphere: From TV Watching to Community Story Telling," the authors describe a web-based collaborative video content workspace for members of a community to compose and share video stories, using desktop or mobile devices. Storisphere is well aligned with the evolution of user-generated content moving into socially connected storytelling that is emerging as a strong current to recreate communities around video content.

In the next two articles, "A Survey of Channel Switching Schemes for IPTV" and "Mitigating IPTV Zapping 
Delay," the authors rightly recognize the importance channel switching time has on the user experience of IPTV systems and over-the-top services. In the current evolution toward "content changing" - with access to many different types of content and the availability of powerful search and recommendation engines - the reduction of the delay when switching from one program to another across providers will become essential, as the "channels" of the future will not be in the hundreds but most likely almost boundless. Both articles review the infrastructure and network challenges that impair channel change, and provide solutions that could be applied.

The Guest Editors would like to thank all the authors for submitting their work for this FT, and all the reviewers for providing their timely reviews. We would also like to thank past Editor-in-Chief Dr. Steve Gorshe for approving this FT and the current Editor-in-Chief Dr. Sean Moore for his promotion of the FT and his continuing support during the review process. Finally, we also thank the IEEE Communication Magazine production staff for making this FT possible.

\section{BIOGRAPHIES}

OSCAR MARTINEZ-BONASTRE [SM] (ombonastre@ieee.org) received his Ph.D. degree in telecommunication engineering at the Polytechnic University of Valencia, Spain. He is an assistant professor and deputy director of international relations at Miguel Hernandez University, Elche, Spain. He is also a contributor to the Operations Research Centre located at the same university. His research interests include the development of congestion control, reliable and layered multicast techniques for multimedia, and new solutions for IPTV and social TV content distribution.

MARIE-JOSÉ MONTPETIT [SM] (mariejo@mit.edu) is an internationally recognized social TV, video, and wireless innovator with over 15 years' experience in architecture, and technologies. She received a Ph.D. in electrical and computer engineering from the Ecole Polytechnique of Montreal. She is currently a lecturer on the future of TV at the MIT Media Laboratory and an advisor to local Boston video and television startups. Her cross-platform IPTV project won the Motorola Innovation Award in 2007, and her work on social TV was rewarded with an MIT Tech Review, TR '10, in 2010. In 2012 Appmarket.tv named her one of the 20 top visionaries in second screen television.

PABLO CESAR (P.S.Cesar@cwi.nl) is a researcher in interactive systems at Centrum Wiskunde \& Informatica (CWI) in the Netherlands. He received his Ph.D. from the Helsinki University of Technology in 2006. He has (co)authored over 50 articles (conference papers and journal articles) about multimedia systems and infrastructures, social multimedia, multimedia content modeling, and user interaction. He has been active in a number of European projects such as Passepartout, SPICE, iNEM4U, TA2, Reverie, and Vconect. He has given tutorials about multimedia systems in prestigious conferences such as ACM Multimedia and the WWW Conference, and is coeditor of the book Social Interactive Television: Immersive Shared Experiences and Perspectives (2009). 